\title{
A New Application of a Fuzzy Linguistic Quality Evaluation System in Digital Libraries
}

\author{
I.J. Pérez and E. Herrera-Viedma \\ Dept. of Computer Science and A.I \\ University of Granada \\ Granada, Spain \\ Email: ijperez,viedma@decsai.ugr.es
}

\author{
J. López-Gijón \\ Dept. of Library Sciences \\ University of Granada \\ Granada, Spain \\ Email: jgijon@ugr.es
}

\author{
F.J. Cabrerizo \\ Dept. of Software Engineering \\ Distance Learning University of Spain (UNED) \\ Madrid, Spain \\ Email: cabrerizo@issi.uned.es
}

\begin{abstract}
In this contribution, we present a new application based on fuzzy linguistic information to evaluate the quality of digital libraries. The quality evaluation of digital libraries is defined using users' perceptions on the quality of digital services provided through their Web sites. We assume a fuzzy linguistic modeling to represent the users' perception and apply automatic tools of fuzzy computing with words based on some weighted aggregation operators to compute global quality evaluations of digital libraries.
\end{abstract}

Keywords-Quality evaluation; Digital Libraries; Fuzzy linguistic modeling.

\section{INTRODUCTION}

The explosive growth of the World Wide Web stimulates the development of fast and effective automated systems that support an easy and effective access to the information relevant to specific users' needs. Digital libraries [1] are one of these automated systems.

Since 1990s, the Internet and the Web has become the primary platform for libraries to build and deliver information resources, services and instructions. Nowadays, in the digital age, we find two kinds of library user information services [1]: i) Traditional library user information services, which are based on a face-to-face personal communication and are developed on-site and ii) Electronic library user information services, which are based on the Web, can be developed onsite or off-site, and are accessible without any geographic and time limitations.

Depending on the library framework, both services are necessary and complementary to develop library activities. However, electronic services allow us to improve the efficiency of the libraries and, therefore, we find hybrid libraries [14] that keep some traditional services but with a great tendency to create new digital services using all Web possibilities. In this framework, we have to deal with new challenges and key issues if we want to offer quality library services to the users, as for example [1]: role academic libraries, quality information resources, Web instructions and training, new assessment and evaluation methodologies, etc.

As digital libraries become commonplace, as their contents and services become more varied, people expect more sophisticated services from their digital libraries [13]. This emergence of digital libraries calls for the need for the evaluation of digital libraries. Furthermore, the expectations and demands for better service and functionality from these users are increasing. Thus, the importance of quality in digital libraries content and services is higher than ever [4]. In this way, evaluation of digital libraries is an essential component for the design of effective digital libraries [17].

Evaluation is a research activity, and it has both theoretical and practical impact [12]. The objective of digital libraries evaluation is to assess to what extent a digital library meets its objectives and offer suggestions for improvements.

The aim of this contribution is to present an application based on fuzzy linguistic information to evaluate the quality of digital libraries. We assume that the quality of a digital library is measured through users' perceptions on the digital services offered through its Web site. Users are invited to fill in a survey built on the set of subjective criteria. To measure quality, conventional measurement tools used by the customers are devised on cardinal or ordinal scales. Moreover, we use an ordinal fuzzy linguistic modeling [5] to represent the user perceptions and tools of computing with words based on the linguistic aggregation operators LOWA [5] and LWA [6] to compute the quality assessments.

The paper is set out as follows. In Section II, some related works and tools of fuzzy computing with words are discussed. Section III describes the system based on fuzzy linguistic information to evaluate the quality of digital libraries. Section IV shows an example of the application on some digital libraries. Finally, some conclusions are pointed out in Section V.

\section{PRELIMINARIES}

In this section, we review some aspects and related works on evaluation of quality in digital libraries and a fuzzy linguistic approach for computing with words.

\section{A. On Evaluation of Quality in Digital Libraries}

Digital libraries are new and innovative information systems, under constant development and change, and, there- 
fore, evaluation is of critical importance to ensure not only their correct evolution but also their acceptance by the user and application communities. The objective of digital libraries evaluation is to assess to what extent a digital library meets its objectives and offer suggestions for improvements [3]. Digital libraries evaluation has many facets depending on the characteristics and the perspective of the evaluating agent.

Different approaches to evaluate the success of a digital library have been studied [2], [4], [9], [12], [15], [16], [17] involving users, collections, and systems, aimed at identifying generalizable metrics or context specific methods. The most recognized digital libraries evaluation criteria are derived from evaluation criteria for traditional libraries, information retrieval system performance and human-computer interaction [3], [12], [15]. Very few studies actually apply all the digital evaluation criteria to assess a digital library. Many of the studies focus on the evaluation of usability of digital libraries. After reviewing usability tests in selected academic digital libraries, Jeng [9] found that ease of use, satisfaction, efficiency and effectiveness are the main applied criteria. Some of the evaluation studies extend to assess performance, content and services of digital libraries while service evaluation mainly concentrates on digital reference [2]. Other evaluation studies also look into the impact of digital libraries [12].

Little research has investigated user's evaluation of digital libraries, in particular, their criteria and their actual assessment of digital libraries [16], [17]. However, as the success of a digital library depends on the users, the value of digital libraries needs be judged by the users of digital libraries. Therefore, in this contribution, we present a model based on fuzzy linguistic information to evaluate the quality of digital libraries which is defined using users' perceptions on the quality of digital services provided through their Web site.

\section{B. A Fuzzy Linguistic Approach for Computing with Words}

Many problems present fuzzy and vague qualitative aspects (decision making, risk assessment, information retrieval, etc.). In such problems, the information cannot be assessed precisely in a quantitative form, but it may be done in a qualitative one, and thus, the use of a linguistic approach is necessary. The fuzzy linguistic approach is an approximate technique appropriate to deal with fuzzy and vague qualitative aspects of problems. It models linguistic information by means of linguistic terms supported by linguistic variables [19], [20]. These are variables whose values are not numbers but words or sentences in a natural or artificial language.

The ordinal fuzzy linguistic approach [5], [6] is a very useful kind of fuzzy linguistic approach used for modeling the computing with words process as well as linguistic aspects of problems. It facilitates the fuzzy linguistic modeling very much because it simplifies the definition of the semantic and syntactic rules. It is defined by considering a finite and totally ordered label set $S=\left\{s_{i}\right\}, i \in\{0, \ldots, \mathcal{T}\}$ in the usual sense, i.e., $s_{i} \geq s_{j}$ if $i \geq j$, and with odd cardinality. Typical values of cardinality used in the linguistic models are odd values, such as 7 or 9 , where the mid term represents an assessment of "approximately 0.5 ", and the rest of the terms being placed symmetrically around it. For example, we can use the following set of nine labels to provide the user evaluations: $\{N=$ None, $E L=$ Extremely Low, $V L=$ Very Low, $L=$ Low, $M=$ Medium, $H=$ High, $V H=$ Very High, EH = Extremely High, T $=$ Total $\}$.

In any linguistic approach we need management operators of linguistic information [5], [6]. An advantage of the ordinal fuzzy linguistic approach is the simplicity and quickness of its computational model. It is based on the symbolic computation [5], [6] and acts by direct computation on labels by taking into account the order of such linguistic assessments in the ordered structure of linguistic terms.

Usually, the ordinal fuzzy linguistic model for computing with words is defined by establishing (i) a negation operator, (ii) comparison operators based on the ordered structure of linguistic terms, and (iii) adequate aggregation operators of ordinal fuzzy linguistic information. In most ordinal fuzzy linguistic approaches the negation operator is defined from the semantics associated to the linguistic terms as $\operatorname{Neg}\left(s_{i}\right)=s_{j} \mid j=\mathcal{T}-i$; and there are defined two comparison operators of linguistic terms:

1) Maximization operator: $M A X\left(s_{i}, s_{j}\right)=s_{i}$ if $s_{i} \geq s_{j}$.

2) Minimization operator: $\operatorname{MIN}\left(s_{i}, s_{j}\right)=s_{i}$ if $s_{i} \leq s_{j}$.

In the following, we present two aggregation operators based on symbolic computation to complete the ordinal linguistic computational model.

1) The LOWA Operator: An important aggregation operator of ordinal linguistic values based on symbolic computation is theLinguistic Ordered Weighted Averaging (LOWA) operator [5]. The LOWA is an operator used to aggregate non-weighted ordinal linguistic information, i.e., linguistic information values with equal importance.

2) The LWA Operator: Another important aggregation operator of ordinal linguistic values is the Linguistic Weighted Averaging (LWA) operator [6]. It is based on the LOWA operator and is defined to aggregate weighted ordinal fuzzy linguistic information, i.e., linguistic information values with not equal importance.

We should point out that the LOWA and LWA operators are the basis of the new fuzzy linguistic evaluation model of digital libraries that we present in this contribution.

\section{A Quality Evaluation System in Digital LIBRARIES}

We use the information quality framework [8] defined in the context of management information systems to evaluate the quality of digital libraries. It has been satisfactorily 
applied to previous quality models for personal Web sites, mobile Internet services and Web sites that store Web documents [7]. In this information quality framework is established that the quality of the information systems cannot be assessed independently of the information consumers' opinions (people who use information). This framework defines four major quality dimensions [8]:

1) Intrinsic quality. This dimension addresses the very nature of the information. It assumes that information has its own quality. The main criterion of the intrinsic quality is the accuracy of the information. If a reputation for inaccurate information becomes common knowledge for a particular information system, this system is viewed as having little added value and will result in a reduction of use. Other criteria of this dimension are: believability, reputation and objectivity.

2) Contextual quality. This dimension emphasizes the importance of the informative aspects of information but from a task perspective. It highlights the requirement that information quality must be considered within the context of the task in hand; it must be relevant, timely, complete, and appropriate in terms of amount, so as to add value to the tasks for which the information is provided. Therefore, some criteria of this dimension are: value-added, relevance, completeness, timeliness, and appropriate amount.

3) Representational quality. This dimension emphasizes the importance of the technical aspects of the (computer-based) structure of the information. It requires information systems to present their information in such a way that it is interpretable, easy to understand, easy to manipulate, and is represented concisely and consistently. Therefore, some of its criteria are: understandability, interpretability, concise representation, and consistent representation.

4) Accessibility quality. This dimension emphasizes the importance of the technical aspects of computer systems that provided access to information. It requires the information system to be accessible but secure. Therefore, among the criteria of this dimension are: accessibility and secure access.

We adapt this information quality framework to develop our evaluation system of the quality of digital libraries. However, as it is oriented to users because the user participation in the quality evaluation processes of services is fundamental to correctly draw the situation of the service, we are going to define a low number of subjective criteria being easily understandable by the users in order to they do not cause the rejection of the users.

Taking into account these considerations, our application is focused on digital services provided through their Web sites. This system presents two elements: (i) a evaluation scheme that contains the subjective criteria and (ii) a com- putation method to generate quality assessments of digital libraries.

3) Evaluation Scheme to Characterize the Quality in Digital Libraries: According to the quality framework [7], [8], we develop an evaluation scheme for evaluating the quality of digital libraries. This evaluation scheme is based both on technical criteria of digital libraries design and on criteria related to the content of information of digital libraries. These criteria are assessed subjectively by users who occasionally visit the digital libraries because they find something that satisfies their information needs.

The evaluation scheme proposed presents the following characteristics:

- It is user driven rather than designed driven. We want to evaluate the quality of digital libraries from the evaluations provided by the different users of them. Therefore, the evaluation scheme should be user driven rather than designed driven from two perspectives:

- Qualitative perspective: The evaluation scheme necessarily requires the inclusion of criteria easily understandable to any user (e.g., relevance, understandability) rather than criteria that can be measured objectively independently of users (ratio of digital journals) or only perceptible by the designers (e.g., code quality or design).

- Quantitative perspective: The evaluation scheme should not include an excessive number of quality criteria in order to help users in understanding it and avoiding confusion. Furthermore, long and complex evaluation schemes cause user idleness and limit their own application possibilities.

- It is weighted: i.e., its quality criteria are not equally important. The quality criteria of the evaluation scheme do not play equal roles in measuring the information quality of a digital library: i.e., some criteria should be more influential than others. For example, user opinions on the information quality of digital libraries (e.g., coverage of the digital library about search topics) must be an important criterion of the evaluation scheme.

We define a user driven and weighted evaluation scheme of digital libraries that contemplates the following four quality dimensions together with their digital quality criteria:

1) Intrinsic quality of digital libraries: To evaluate the intrinsic quality or accuracy of digital libraries, we define the following subjective criterion: you find what you are looking for.

2) Contextual quality of digital libraries: To evaluate the information quality of the digital libraries within the context, the following subjective criteria are defined: coverage of the digital library about search topics, information electronic services about new inputs, added value information profits and also global satisfaction degree. 
3) Representational quality of digital libraries: It is evaluated taking into account the following subjective criteria: understandability of the digital library Web site and training received.

4) Accessibility and interaction quality of digital libraries: It is measured considering the following subjective criteria: variety of search tools, navigability of the digital library Web site, satisfaction degree with the computing infrastructure and satisfaction degree with the response time.

4) Computation Method to Generate Quality Assessments in Digital Libraries: The computation method to generate quality assessments in digital libraries is like a multi-person multi-criteria decision making method in which the search alternatives are digital libraries. In a multi-criteria decision making method, the goal consists of searching the best alternatives according to the assessments provided by a group of experts with respect to a set of evaluation criteria [10]. To do that, through the aggregation of the experts' assessments the quality of alternatives is measured and, later, the exploitation of those quality values leads to the selection of the best alternatives. In our case, the goal consists of computing quality evaluations of digital libraries in order to select the digital library that could better meet the user information needs, but as in a multi-criteria decision context, we compute those values according to the assessments provided by a group of persons (digital libraries users).

As it is known, in multi-criteria decision making processes the chosen aggregation operator is a critical aspect that has a direct influence on the success of the decision process. The quantifier guided aggregation operators based on the OWA operator constitute a successful tool to aggregate information because of its flexibility: i.e., it allows representation in the aggregations of different interpretations of the concept of majority by means of the fuzzy linguistic quantifier [18]. We do the same in our computation method.

We have designed a computation method to generate quality assessment in digital libraries that has two main characteristics:

- It is a user centered computation method. The quality assessment in digital libraries is obtained from individual linguistic judgments provided by digital libraries users rather than from assessments obtained objectively by means of the direct observation of the digital libraries characteristics.

- It is a majority guided computation method. The quality assessments are values representative of the majority of individual judgments provided by the digital libraries users. The aggregation to compute the quality assessments is developed by means of the LOWA and LWA operators.

Firstly, we define a quality evaluation questionnaire providing questions for each one of the subjective criteria proposed in the evaluation scheme, i.e., there are eleven questions: $\left\{q_{1}, \ldots, q_{11}\right\}$. For example, for the subjective criterion you find what you are looking for, the question $q_{1}$ can be: "What is the degree in which you usually find what you are looking for?". The quality evaluation questionnaire can be as follows:

1) Question 1: "What is the degree in which you usually find what you are looking for?".

2) Question 2: "What is the coverage degree of the digital library about search topics?".

3) Question 3: "What is the degree of information electronic service about new inputs?".

4) Question 4: "What is the degree of added value information profits?".

5) Question 5: "What is your global satisfaction degree?".

6) Question 6: "What is the understandability degree of the digital library Web site?".

7) Question 7: "What is the degree of training received?".

8) Question 8: "What is the degree of variety of search tools?".

9) Question 9: "What is the navigability degree of the digital library Web site?".

10) Question 10: "What is your satisfaction degree with the computing infrastructure?".

11) Question 11: "What is your satisfaction degree with the response time?".

The concept behind each question is rated on a linguistic term set $S$. To do so, we can use the set of linguistic terms proposed in Sec. II-B to rate all the questions. We use fuzzy linguistic variables to represent users' opinions by mean of linguistic labels because they are more easily understood by the users than numerical ones. In addition, we assume that each subjective criteria does not have the same importance in the evaluation scheme, i.e., it is assigned a relative linguistic importance degree for each subjective criterion: $\left\{I\left(q_{1}\right), \ldots, I\left(q_{11}\right)\right\}, I\left(q_{i}\right) \in S$. These importance degree could be obtained from a set of experts or users' judgements.

Then, assuming that we have a group of users, $\left\{e_{1}, \ldots, e_{L}\right\}$, that have filled in the questionnaire, and given a digital library, $\mathcal{A}_{m}$, the computation method generates its quality assessment, $r^{m} \in S$, using the linguistic aggregation operators LOWA and LWA in the following steps:

- Calculate for each subjective criterion, $q_{i}$, the global quality assessment, $r_{i}^{m} \in S$, by aggregating the evaluation judgments provided by the group of users on the subjective criterion by means of the LOWA operator $\phi$ :

$$
r_{i}^{m}=\phi_{Q}\left(e_{1}\left(q_{i}\right), \ldots, e_{L}\left(q_{i}\right)\right),
$$

where $e_{l}\left(q_{i}\right) \in S$ is the linguistic preference provided by the $e_{l}$ on subjective criteria represented by the question $q_{i}$. Therefore, $r_{i}^{m}$ is a linguistic measure that represents the quality assessment of the digital library 
$\mathcal{A}_{m}$ with respect to subjective criterion $q_{i}$ according to the majority (represented by the fuzzy linguistic quantifier $Q$ ) of linguistic evaluation judgments provided by the group of users $\left\{e_{1}, \ldots, e_{L}\right\}$.

- Calculate for the digital library, $\mathcal{A}_{m}$, its quality assessment, $r^{m} \in S$, by aggregating its individual quality assessment, $r_{i}^{m} \in S$, for each subjective criterion, $q_{i}$, by means of the LWA operator $\Phi$ :

$$
r^{m}=\Phi_{Q}\left(\left(I\left(q_{1}\right), r_{1}^{m}\right), \ldots,\left(I\left(q_{11}\right), r_{11}^{m}\right)\right) .
$$

In this case, $r^{m}$ is a measure that represents the quality assessment of the digital library $\mathcal{A}_{m}$ according to the majority (represented by the fuzzy linguistic quantifier $Q$ ) of linguistic evaluation judgments provided by the group of users about important subjective criteria $q_{i}$.

\section{Application to Evaluate Digital Libraries}

In this section, we present an example of application where the evaluation system is used to evaluate the quality of three Spanish academic digital libraries:

1) Digital library of Jaén University.

2) Digital library of Córdoba University.

3) Digital library of Málaga University.

The reason for the selection of academic digital libraries is due to they are the first kind of libraries that are getting most benefit from the Web possibilities to help in teaching, learning and researching activities [11].

Fifty subjects were recruited for this study. They were $\mathrm{Ph}$. D. students from University of Granada who were interested in the learning and using of digital libraries. Female $(56 \%)$ and male (44\%) subjects are pretty close in the composition of the subject pool. All of them had enough knowledge of digital libraries, and have used and searched digital libraries before this study. Two reasons were considered for the recruitment:

- These subjects have a need to understand digital libraries and have some experience with the use of digital libraries.

- These subjects are the targeted audience for similar types of digital libraries. They are users of the digital library of Granada University.

The best way to evaluate digital libraries is to actually use them. Fifty subjects tried to find information related to six questions for each one of the three academic digital libraries selected for this study. For example, subjects were instructed to find a book titled "Fuzzy set theory and its applications" and its authors. In another question, subjects need to identify two approaches to find information about L. A. Zadeh and five papers of him. The subjects could work on the digital libraries at any locations that they felt comfortable.

To obtain the judgements supplied by the users for each academic digital library, we use the quality evaluation questionnaire presented in Sec. III. It is composed of the eleven queries, one for each subjective criterion $q_{i}$. Furthermore, the set of nine labels proposed in Sec. II-B is used to provide the user evaluations. In Table I, we can see the global quality assessment, $r_{i}^{m}=\phi_{Q}\left(e_{1}\left(q_{i}\right), \ldots, e_{50}\left(q_{i}\right)\right) \in S$, for each subjective criterion $q_{i}$ for each academic digital library selected for this study.

Table I

GLOBAL QUALITY ASSESSMENT, $r_{i}^{m} \in S$, FOR EACH SUBJECTIVE CRITERION $q_{i}$.

\begin{tabular}{|c|c|c|c|}
\hline & Jaén & Málaga & Córdoba \\
\hline$q_{1}$ & $V H$ & $E H$ & $V H$ \\
\hline$q_{2}$ & $V H$ & $E H$ & $H$ \\
\hline$q_{3}$ & $L$ & $L$ & $L$ \\
\hline$q_{4}$ & $M$ & $M$ & $M$ \\
\hline$q_{5}$ & $V H$ & $V H$ & $H$ \\
\hline$q_{6}$ & $V H$ & $V H$ & $V H$ \\
\hline$q_{7}$ & $M$ & $H$ & $M$ \\
\hline$q_{8}$ & $H$ & $M$ & $H$ \\
\hline$q_{9}$ & $E H$ & $V H$ & $M$ \\
\hline$q_{10}$ & $E H$ & $E H$ & $V H$ \\
\hline$q_{11}$ & $V H$ & $H$ & $H$ \\
\hline
\end{tabular}

In this particular example, we can see that the subjective criterion $q_{3}$ has a lower evaluation in the three academic digital libraries, that is, users think that the information electronic services about new inputs should be improved. In addition, the subjective criterion $q_{6}$ has a higher evaluation in the three academic digital libraries, in fact, its evaluation is near to the maximum one, which means that almost every user agrees on the good evaluation of the understandability degree of the three digital libraries Web sites studied. On the other hand, subjective criteria $q_{9}$ has a much better evaluation for the academic digital library of Jaén than for the academic digital library of Córdoba, which means that the navigability of the digital library Web site should be improved in the academic digital library of Córdoba.

Assuming the linguistic importance degrees $\{E H, E H, M, M, T, H, M, M, H, M, M\} \quad$ associated with the quality criteria, the linguistic quantifier most of defined as $Q(r)=r^{1 / 2}$, and using the LWA operator, in Table II, we can see the quality assessment, $r^{m}=\Phi_{Q}\left(\left(I\left(q_{1}\right), r_{1}^{m}\right), \ldots,\left(I\left(q_{11}\right), r_{11}^{m}\right)\right) \in S$, for each academic digital library selected for this study.

Table II

QUALITY ASSESSMENT, $r^{m} \in S$, FOR EACH ACADEMIC DIGITAL LIBRARY.

\begin{tabular}{|c|c|c|c|}
\hline & Jaén & Málaga & Córdoba \\
\hline$r^{m}$ & $H$ & $H$ & $M$ \\
\hline
\end{tabular}

For example, the quality assessment for the academic digital library of Jaén is obtained from the following expression:

$$
\begin{aligned}
& r^{J a e n}=\Phi_{Q}((E H, V H),(E H, V H),(M, L),(M, M), \\
& (T, V H),(H, V H),(M, M),(M, H),(H, E H),(M, E H), \\
& (M, V H))=H .
\end{aligned}
$$


To develop this expression it is necessary to calculate the weighting vector $W$. To do so, we make use of the linguistic quantifier most of defined as $Q(r)=r^{1 / 2}$ :

$$
\begin{gathered}
Q(0)=0, \quad Q(1 / 11)=0.30, \quad Q(2 / 11)=0.43, \\
Q(3 / 11)=0.52, \quad Q(4 / 11)=0.60, \quad Q(5 / 11)=0.67, \\
Q(6 / 11)=0.74, \quad Q(7 / 11)=0.80, \quad Q(8 / 11)=0.85, \\
Q(9 / 11)=0.90, \quad Q(10 / 11)=0.95, \quad Q(1)=1
\end{gathered}
$$

And we obtain the following weighting vector: $W=$ $(0.30,0.13,0.09,0.08,0.07,0.07,0.06,0.05,0.05,0.05,0.05)$

As $\operatorname{orness}(W)=0.67 \geq 0.5$, then $h=\operatorname{MIN}\left(c_{i}, a_{i}\right)$, and therefore:

$$
\begin{aligned}
& \Phi_{Q}((E H, V H),(E H, V H),(M, L),(M, M),(T, V H), \\
& (H, V H),(M, M),(M, H),(H, E H),(M, E H),(M, V H)) \\
& =\phi_{Q}(V H, V H, L, M, V H, H, M, M, H, M, M) \\
& =W \cdot B^{T}=C^{11}((V H, 0.30),(V H, 0.13),(V H, 0.09), \\
& (H, 0.08),(H, 0.07),(M, 0.07),(M, 0.06),(M, 0.05), \\
& (M, 0.05),(M, 0.05),(L, 0.05))=0.30 \odot V H \oplus 0.70 \odot \\
& C^{10}((V H, 0.19),(V H, 0.13),(H, 0.11),(H, 0.10),(M, 0.10), \\
& (M, 0.08),(M, 0.07),(M, 0.07),(M, 0.07),(L, 0.07))=H .
\end{aligned}
$$

\section{CONCLUSIONS}

The user satisfaction is essential for the success of a digital library. In this way, we have presented a new application based on fuzzy linguistic approach to evaluate the quality of digital libraries, which is defined using users' perceptions on the quality of digital services provided through their Web sites. This application is user oriented because it only considers user evaluation judgments to evaluate the quality of digital libraries.

\section{ACKNOWLEDGMENT}

This paper has been developed with the financing of FEDER funds in FUZZYLING project (TIN2007-61079), PETRI project (PET2007-0460), project of Ministry of Public Works (90/07) and Excellence Andalusian Project (TIC5299).

\section{REFERENCES}

[1] D. Bawden and P. Vilar, Digital libraries: To meet or manage user expectations, Aslib Proceedings: New Information Perspectives 58(4) (2006) 346-354.

[2] D. S. Carter and J. Janes, Unobtrusive data analysis of digital reference questions and service at the Internet Public Library: An exploratory study, Library Trends 49(2) (2000) 251-265.

[3] G. G. Chowdhury and S. Chowdhury, Introduction to Digital Libraries (Facet Publisher, London, 2003).

[4] N. Fuhr, G. Tsakonas, T. Aalberg, M. Agosti, P. Hansen, S. Kapidakis, et al., Evaluation of digital libraries, International Journal on Digital Libraries 8(1) (2007) 21-38.
[5] F. Herrera, E. Herrera-Viedma and J. L. Verdegay, Direct approach processes in group decision making using linguistic OWA operators, Fuzzy Sets and Systems 79(2) (1996) 175190.

[6] F. Herrera and E. Herrera-Viedma, Aggregation operators for linguistic weighted information, IEEE Transactions on Systems, Man and Cybernetycs, Part A: Systems and Humans 27(5) (1997) 646-656.

[7] E. Herrera-Viedma, G. Pasi, A. G. López-Herrera and C. Porcel, Evaluating the information quality of web sites: A methodology based on fuzzy computing with words, Journal of American Society for Information Science and Technology 57(4) (2006) 538-549.

[8] K. Huang, Y. W. Lee and R. Y. Wang, Quality Information and Knowledge (Prentice Hall, Upper Saddle River, NJ, 1999).

[9] J. Jeng, Usability assessment of academic digital libraries: Effectiveness, efficiency, satisfaction, and learnability, LIBRI 55(2-3) (2005) 96-121.

[10] M. Köksalan and C. Tuncer, A dea-based approach to ranking multi-criteria alternative, International Journal of Information Technology \& Decision Making 8(1) (2009) 29-54.

[11] G. Marchionini and H. Maurer, The roles of digital libraries in teaching and learning, Communication of ACM 38(4) (1995) $67-75$.

[12] G. Marchionini, Evaluation digital libraries: A longitudinal and multifaceted view, Library Trends 49(2) (2000) 304-333.

[13] M. E. Renda and U. Straccia, A personalized collaborative digital library environment: A model and an application, Information Processing and Management 41(1) (2005) 5-21.

[14] C. Rushbridge, Towards the hybrid library, D-Lib Magazine July/August (1998).

[15] T. Saracevic, Digital library evaluation: Toward an evolution of concepts, Library Trends 49(3) (2000) 350-369.

[16] H. I. Xie, Evaluation of digital libraries: Criteria and problems from user's perspectives, Library and Information Science Research 28(3) (2006) 433-452.

[17] H. I. Xie, Users' evaluation of digital libraries (DLs): Their uses, their criteria, and their assessment, Information Processing and Management 44(3) (2008) 1346-1373.

[18] R. R. Yager, On ordered weighted averaging aggregation operators in multicriteria decision making, IEEE Transactions on Systems, Man and Cybernetycs 18(1) (1988) 183-190.

[19] L. A. Zadeh, The concept of a linguistic variable and its applications to approximate reasoning. Part I, Information Sciences 8(3) (1975) 199-249.

[20] L. A. Zadeh, A computational approach to fuzzy quantifiers in natural languages, Computer \& Mathematics with Applications 9(1) (1983) 149-184. 